\title{
Uso de níveis crescentes de concentrado extrusado e silagem de milho na dieta de ovinos
}

\author{
Gustavo Roberto Dias Rodrigues ${ }^{1}$; Marco Túlio Santos Siqueira ${ }^{2}$; Erica Beatriz Schultz ${ }^{3 *}$; Karla Alves Oliveira ${ }^{4}$; \\ Luciano Fernandes Sousa ${ }^{5}$; Gilberto de Lima Macedo Júnior ${ }^{6}$
}

DOI: https://doi.org/10.35699/2447-6218.2021.33741

\begin{abstract}
Resumo
Objetivou-se avaliar os efeitos de diferentes relações concentrado:volumoso, com a utilização de concentrado extrusado e silagem milho, sobre o consumo, digestibilidade, comportamento ingestivo e metabolismo de ovinos. Foram utilizadas 20 ovelhas Santa Inês com peso de $50 \mathrm{~kg}$ e idade média três anos distribuídas em delineamento inteiramente casualizado. Os tratamentos avaliados tiveram diferentes relações concentrado: volumoso, com a utilização do concentrado extrusado comercial Beef Agnus ${ }^{\circledR}$ (B) (Nuttrata Nutrição Animal, Itumbiara-GO, Brasil) e Silagem de milho (S). Os tratamentos consistiram: 40\% de Beef Agnus ${ }^{\circledR}$ e 60\% de Silagem de milho (40\%B:60\%S); 50\% Beef Agnus ${ }^{\circledR}$ e 50\% de Silagem de milho (50\%B:50\%S); 60\% de Beef Agnus ${ }^{\circledR}$ e 40\% de Silagem de milho (60\%B:40\%S); $70 \%$ de Beef Agnus $^{\circledR}$ e 30\% de Silagem de milho (70\%B:30\%S). As análises de regressão foram realizadas a 5\% de probabilidade para erros do tipo I. O consumo de matéria seca (CMS), CMS em relação ao peso corporal, CMS em relação ao peso metabólico e eficiências de ruminação e mastigação obtiveram resposta linear negativa ao aumento da inclusão de silagem de milho $(\mathrm{P}<0,05)$. A glicemia por horário de colheita e as proteínas totais apresentaram resposta quadrática $(\mathrm{P}<0,05)$. A digestibilidade, os parâmetros urinários e fecais e os metabolitos energéticos se mantiveram a redução da proporção de volumoso na dieta $(\mathrm{P}>0,05)$. Conclui-se que a utilização de concentrado extrusado promove maior consumo aos animais e não gera danos ao comportamento ingestivo, digestibilidade e metabolismo de ovinos.
\end{abstract}

Palavras chave: Extrusão. Ruminantes. Silagem de milho. Ovis aries.

\section{Use of increasing levels of extruded concentrate and corn silage in sheep diet}

\begin{abstract}
:
The aim was to evaluate the effects of different concentrate: roughage relationships, using extruded concentrate and corn silage, on the intake, digestibility, ingestive behavior, and metabolism of sheep. Twenty Santa Inês ewes with a weight of $50 \mathrm{~kg}$ and an average age of three years were used, assigned in a completely randomized design. The evaluated treatments had different concentrate: roughage ratios, using the commercial extruded concentrate Beef Agnus ${ }^{\circledR}$ (B) (Nuttrata Animal Nutrition, Itumbiara-GO, Brazil) and Corn silage (S). The treatments consisted of: 40\% Beef Agnus ${ }^{\circledR}$ and 60\% Corn silage (40\% B: 60\% S); 50\% Beef Agnus® and 50\% Corn silage (50\% B: 50\% S); $60 \%$ Beef Agnus ${ }^{\circledR}$ and $40 \%$ Corn silage (60\% B: 40\% S); 70\% Beef Agnus ${ }^{\circledR}$ and 30\% Corn silage (70\% B: 30\% S). The regression analyzes were performed at $5 \%$ probability for type I errors. The dry matter intake (DMI), DMI in
\end{abstract}

${ }^{1}$ Universidade Federal de Uberlândia. Uberlândia, MG. Brasil.

https://orcid.org/0000-0001-9438-3724

${ }^{2}$ Universidade Federal de Uberlândia. Uberlândia, MG. Brasil.

https://orcid.org/0000-0002-2098-8568

${ }^{3}$ Universidade Federal de Viçosa. Viçosa, MG. Brasil.

http://orcid.org/0000-0003-1916-2117

${ }^{4}$ Universidade Estadual Paulista. Jaboticabal, SP. Brasil.

https://orcid.org/0000-0002-7792-2615

${ }^{5}$ Universidade Federal do Tocantins, Araguaína, TO. Brasil. https://orcid.org/0000-0002-6072-9237

${ }^{6}$ Universidade Federal de Uberlândia. Uberlândia, MG. Brasil. https://orcid.org/0000-0001-5781-7917

*Autor para correspondência: ericabeatrizschultz@gmail.com

Recebido para publicação em 22 de Maio de 2021. Aceito para publicação 25 de Junho de 2021 e-ISSN: 2447-6218 / ISSN: 2447-6218. Atribuição CC BY. 
Rodrigues, G. R. D. et al.

relation to body weight, DMI in relation to metabolic weight, rumination, and chewing efficiencies showed a negative linear response to increased inclusion of corn silage $(\mathrm{P}<0.05)$. Glycemia by harvest time and total proteins showed a quadratic response $(\mathrm{P}<0.05)$. Digestibility, urinary and fecal parameters, and energy metabolites were maintained with an reduce in the roughage proportion in the diet $(\mathrm{P}>0.05)$. It is concluded that the use of extruded concentrate promotes the greater intake of the animals and does not cause damage to the ingestive behavior, digestibility, and metabolism of sheep.

Key words: Extrusion. Ruminants. Corn silage. Ovis Aries.

\section{Introdução}

A busca por tecnologias para aumentar a produtividade dos rebanhos, sem onerar os custos de produção, traz destaque positivo para os alimentos extrusados, uma vez que esses produtos atuam na maximização da eficiência digestiva (Mourão et al., 2012). De acordo com Silva et al. (2015b) a extrusão pode ser definida como uma etapa de processamento industrial de matéria prima sólida aplicando altas temperaturas, pressão e umidade no alimento para gerar expansão dos ingredientes, gelatinização do amido, redução de fatores antinutricionais e desnaturação de proteínas.

Alimentos extrusados não só melhoram a digestibilidade, mas também ampliam a biodisponibilidade de nutrientes presentes nas matérias primas (Singh et al., 2010). Na nutrição de ruminantes, esses produtos têm a capacidade de sincronizar o fornecimento das fontes alimentares, uma vez que as proteínas, energia, minerais, aditivos e fibras tornam-se um único alimento. Além disso, ampliam a fermentação ruminal gerando melhor aproveitamento dos alimentos e beneficiam o ambiente ruminal pela manutenção de seu pH (Reddy \& Reddy, 2015).

Em contrapartida, um dos fatores que determinam o consumo de animais ruminantes é a relação concentrado: volumoso. Segundo Macedo Júnior et al. (2012) com aumento na proporção de volumo há também aumento nos níveis de fibra, redução do teor energético da dieta ofertada podendo levar ao efeito de enchimento ruminal e redução no consumo. Já dietas ricas em alimentos concentrados podem ocasionar efeitos negativos à fermentação ruminal e proporcionar distúrbios metabólicos, por exemplo, acidose ruminal (Oetzel, 2017).

Estudos com uso de volumo e concentrado extrusado tem demonstrado efeito positivo no uso desta tecnologia na alimentação de ovinos (Macedo Junior et al., 2019; Ruela et al., 2020). Sendo assim, com a criação de novas tecnologias na nutrição animal e surgimento de novas fontes alimentares na forma processada, as avaliações de consumo, digestibilidade, comportamento ingestivo e curva glicêmica são fundamentais para inferências acerca das características positivas e negativas do uso dos alimentos extrusados, para ruminantes.

Nossa hipótese é de que as diferentes relações concentrado: volumoso, com a utilização de concentrado extrusado, possa melhorar o consumo e digestibilidade dos alimentos, além de parâmetros ruminais e metabólicos. Dessa forma, o objetivo foi avaliar diferentes relações concentrado: volumoso, com a utilização de concentrado extrusado comercial e silagem de milho, sobre o consumo, digestibilidade, comportamento ingestivo, metabólitos sanguíneos e curva glicêmica de ovelhas adultas da raça Santa Inês.

\section{Material e Métodos}

O experimento teve condução no setor de caprinos e ovinos, da fazenda Capim Branco, pertencente à Universidade Federal de Uberlândia (UFU). Sua realização ocorreu durante o mês de janeiro de 2017, com duração total de 20 dias, onde os quinze primeiros dias foram dedicados à adaptação dos animais a dieta experimental e gaiolas metabólicas, e os últimos cinco dias foram destinados para coletas de fezes, urina, sobras de alimento e água. O ensaio contou com a aprovação da Comissão de Ética e Utilização dos Animais (CEUA) da UFU sob o número de protocolo 092/16.

As unidades experimentais utilizadas foram vinte ovelhas da raça Santa Inês, não gestantes, não lactantes, com peso médio de $50 \mathrm{~kg}$ e idade média de três anos, distribuídas em delineamento inteiramente casualizado. Para o início da experimentação, todos os animais foram pesados e vermifugados com Levamisol (via oral) no primeiro dia do experimento e feita observação da mucosa ocular. Em seguida, as ovelhas foram alocadas em gaiolas metabólicas equipadas com comedouro, bebedouro, saleiro, piso ripado e artefato para separação de fezes e urina, de acordo com padrão dos Institutos Nacionais de Ciência e Tecnologia (INCT).

Os grupos avaliados foram constituídos por diferentes relações concentrado: volumoso, com a utilização do concentrado extrusado comercial Beef Agnus ${ }^{\circledR}$ (B) (Nuttrata Nutrição Animal, Itumbiara - GO, Brasil) e Silagem de milho (S). Os tratamentos experimentais foram: 40\% de Beef Agnus ${ }^{\circledR}$ e 60\% de Silagem de milho (40\%B:60\%S); 50\% Beef Agnus ${ }^{\circledR}$ e 50\% de Silagem de milho (50\%B:50\%S); 60\% de Beef Agnus ${ }^{\circledR}$ e 40\% de Silagem de milho (60\%B:40\%S); 70\% de Beef Agnus ${ }^{\circledR}$ e 30\% de Silagem de milho (70\%B:30\%S) (Tabela 1). 
Uso de níveis crescentes de concentrado extrusado e silagem de milho na dieta de ovinos

Tabela 1- Esquema dos grupos experimentais

\begin{tabular}{ccc}
\hline Tratamentos & Beef Agnus & Silagem de milho \\
\hline T1 & $40 \%$ & $60 \%$ \\
T2 & $50 \%$ & $50 \%$ \\
T3 & $60 \%$ & $40 \%$ \\
T4 & $70 \%$ & $30 \%$ \\
\hline
\end{tabular}

A alimentação foi fornecida aos animais duas vezes ao dia, às 08:00hrs e posteriormente às 16:00hrs, sendo ofertado $50 \%$ do total diário em cada um desses turnos. Além disso, a dieta foi balanceada para ter-se entre $5-10 \%$ de sobras do total fornecido. Durante todos os 20 dias do experimento, os animais tiveram livre acesso à água e sal mineral específico para ovinos.
O produto Beef Agnus ${ }^{\circledR}$ é um concentrado proteico-energético extrusado indicado para ovinos. É composto por ureia, sal comum $(\mathrm{NaCl})$, fosfato bicálcico, sulfato de cálcio, cloreto de potássio, calcário calcítico, farelo de algodão, milho (70\% amido), virginiamicina $50 \%$ e núcleo ovinos $8 \%$. As composições químicas do Beef Agnus $^{\circledR}$, da silagem de milho e das dietas experimentais estão descritas nas Tabela 2.

Tabela 2 - Composições percentuais e químicas do Beef Agnus ${ }^{\circledR}$, silagem de milho e das dietas em função dos tratamentos

\begin{tabular}{cccccc}
\hline Alimento & MS (\%) & PB (\%) & FDN (\%) & FDA (\%) & NDT (\%) \\
\hline Beef Agnus ${ }^{\circledR *}$ & 88,10 & 18,20 & 17,00 & 9,90 & 85,20 \\
Silagem de milho** & 31,18 & 6,30 & 55,40 & 32,80 & 64,88 \\
\hline Tratamento & MS (\%) & PB (\%) & FDN (\%) & FDA (\%) & NDT (\%) \\
\hline 40B:60S & 53,95 & 11,06 & 40,04 & 23,64 & 73,01 \\
50B:50S & 59,64 & 12,25 & 36,20 & 21,35 & 75,04 \\
60B:40S & 65,33 & 13,44 & 32,36 & 19,06 & 77,07 \\
70B:30S & 71,02 & 14,63 & 28,52 & 16,77 & 79,10 \\
\hline
\end{tabular}

B: Beef Agnus; S: Silagem de milho; MS: matéria seca; PB: proteína bruta; FDN: fibra em detergente neutro; FDA: fibra em detergente ácido; NDT: nutrientes digestíveis totais; *Valores fornecidos pelo fabricante Nutratta ${ }^{\odot} . * *$ Valores obtidos através de análises química efetuadas no Laboratório de Nutrição Animal da Universidade Federal de Uberlândia.

Ao final dos quinze dias destinados à adaptação dos animais, foram feitas as coletas de dados. Em todos os cinco dias de coleta, todos os alimentos ofertados, sobras e fezes foram pesados e amostrados por meio da utilização de uma balança eletrônica de precisão de cinco gramas. Para realização das análises químicas e cálculo de consumo e digestibilidade da matéria seca, foram utilizadas amostras compostas provenientes das amostras simples de cada animal. Após o período de coleta, estas amostras foram armazenadas em freezers horizontais a uma temperatura de $-15^{\circ}$ para conservação das mesmas, e em seguida, os alimentos ofertados, sobras e fezes foram pré-secos em estufa ventilada à $55^{\circ} \mathrm{C}$ durante 72 horas, até a verificação de peso constante das amostras. Em seguida, foram trituradas em um moinho de facas do tipo Willey (ABNT), em partículas de $1 \mathrm{~mm}$. As amostras foram levadas ao Laboratório de Nutrição Animal da UFU, onde foi possível realizar a matéria seca definitiva das porções de ofertado, sobras e fezes, em estufa à $105^{\circ} \mathrm{C}$ durante 24 horas. Sendo realizado os cálculos de consumo e digestibilidade da matéria seca de acordo com Maynard et al. (1984).
A urina dos animais foi coletada por meio da utilização de baldes com telas, para que ocorresse a retenção das fezes que foram recolhidas em bandejas plásticas. A mensuração do volume urinário contou com o uso de provetas graduadas de plástico, com capacidade de dois litros e precisão de $20 \mathrm{~mL}$. Quantificou-se o volume de urina excretado por cada animal durante o período de 24 horas, sendo amostrado $20 \%$ do total diário de todos os baldes coletores em cada um dos cindo dias de coleta. Em seguida, essas amostras foram filtradas com a utilização de filtros descartáveis de papel e armazenadas em garrafas plásticas identificadas mantidas em um freezer à $-15^{\circ} \mathrm{C}$ para futuras análises.

Com relação à densidade de urina, foi utilizado um refratômetro manual portátil Megabrix ${ }^{\circledR}$ (Fremont, Ohio, Estados Unidos) para mensuração dessa variável. Com o auxílio de pipetas descartáveis, $1 \mathrm{~mL}$ de urina foi transferido do balde coletor de cada animal para o prisma do optômetro. Esse procedimento realizado sob luz fluorescente sempre na mesma posição. Entre a mensuração de cada amostra, o refratômetro foi higienizado 
Rodrigues, G. R. D. et al.

e seco com papel toalha para evitar interferências entre os resultados avaliados.

O escore fecal foi avaliado de acordo com a escala descrita por Gomes et al., (2012), onde na escala um (1) as fezes são ressecadas e sem brilho; na escala dois (2) as fezes são normais; na escala três (3) as fezes são ligeiramente amolecidas; na escala quatro (4) as fezes são amolecidas, perdendo o formato e coladas umas às outras; na escala cinco (5) as fezes são amolecidas e sem formato normal; e na escala seis (6) as fezes são diarreicas.

O cálculo da ingestão de água pelos animais foi realizado por meio da diferença entre a quantidade de água ofertada diariamente e as sobras mensuradas no dia seguinte, levando em consideração a quantidade de água que evaporou. Utilizou-se uma proveta graduada de plástico com capacidade de dois litros e exatidão de $20 \mathrm{~mL}$ para identificação desses valores. Durante todos vinte dias do ensaio experimental ofertou-se uma quantidade padrão de seis litros de água para cada animal, sendo que caso houvesse necessidade, fornecia-se quantidades maiores. A evaporação foi mensurada a cada 24 horas por meio da inserção de um balde contendo seis litros de água no galpão experimental, em local de acesso restrito aos animais. O balde de evaporação foi alocado durante o momento de fornecimento de água para os animais, em uma superfície plana correspondente à altura dos baldes presentes nas gaiolas metabólicas. Os níveis de água evaporada também foram obtidos pela diferença entre os seis litros ofertados e suas sobras verificadas no dia seguinte. Para a realização dos cálculos a quantidade total de água evaporada foi descontada do consumo diário de água de cada animal.

Durante o quinto dia do período de coleta, ocorreu a mensuração do comportamento ingestivo, de acordo com a metodologia proposta por Fischer et al. (1998) onde os animais foram avaliadas a cada cinco minutos, onde observava-se se estavam ingerindo alimento ou água separadamente (ING), ruminando (RUM) ou em ócio (ÓCIO).

Os cálculos das atividades foram feitos em minutos por dia, admitindo que, nos cinco minutos subsequentes a cada observação, o animal permaneceu na mesma atividade. O tempo total gasto em mastigação (MAST) foi obtido pela soma entre o tempo gasto em ingestão (ING) e ruminação (RUM). As eficiências de ingestão (Eing), mastigação (Emast) e ruminação (Erum) foram obtidas segundo Polli et al. (1996), de acordo com as equações:

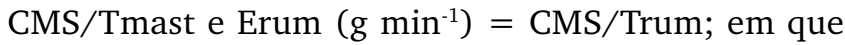
CMS é consumo de MS ( $\mathrm{g}$ MS dia-1), Tal é o tempo de alimentação (min dia $^{-1}$ ), Tmast é o tempo em mastigação (min dia $^{-1}$ ) e Trum é o tempo em ruminação (min dia $\left.{ }^{-1}\right)$.

As coletas de sangue para avaliação dos componentes bioquímicos também foram realizadas após os quinze dias do período de adaptação dos animais, sendo feitas no primeiro, terceiro e quinto dia de coleta do experimento, antes da primeira refeição ofertada no dia com os animais em jejum desde a última refeição disponibilizada no dia anterior. As colheitas de sangue ocorreram por venopunção da jugular com tubos Vacutainer ${ }^{\circledR}$ (BD) sem anticoagulante. Os componentes bioquímicos avaliados para determinação do metabolismo energético foram: triglicerídeos, colesterol e frutosamina; e para determinação do metabolismo proteico foram: ureia, proteínas totais, ácido úrico, albumina e creatinina.

As colheitas para avaliação glicêmica ocorreram no último dia de coleta de dados em horários pré-definidos, sendo às 08:00 (antes da primeira refeição), 11:00, 14:00, 17:00 e 20:00 horas. Excepcionalmente nesse dia, a segunda refeição somente foi ofertada após o recolhimento das 20:00 horas. As amostras destinadas à análise de glicemia foram obtidas por venopunção da jugular com a utilização de tubos Vacutainer ${ }^{\circledR}$ contendo fluoreto e EDTA, identificados para cada animal.

Todas as amostras de sangue coletadas passaram por uma centrifuga à 3000 rotações por minuto, durante 10 minutos. O soro foi separado em alíquotas que foram armazenados em freezers verticais à $-15^{\circ} \mathrm{C}$ para futuras análises laboratoriais. $\mathrm{O}$ processamento dessas amostras foi realizado com a utilização de um analisador bioquímico automatizado Bioplus 2000 (Bioplus $^{\circledR}$, Barueri-SP, Brasil), usando kit comercial da Lab Test Diagnósticos $\mathrm{S}$. $\mathrm{A}^{\odot}$ (Labtest Diagnóstica S. A., Lagoa Santa-MG, Brasil).

Utilizou-se delineamento inteiramente casualizado, sendo para a glicemia ao longo do dia foi considerado delineamento inteiramente com medidas repetidas no tempo. Esta variável foi testada para condição de esfericidade, que não foi aceita. Portanto, utilizou-se a análise de modelos mistos, em que foram avaliadas todas as estruturas de covariâncias (S) disponíveis no pacote do software SAS (SAS Institute, 2012) que modelam a dependência dos erros do modelo. Para selecionar a estrutura de covariância que melhor explique a correlação residual, foi utilizado o critério de informação de Akaike (AIC), sendo escolhida, para cada variável, a estrutura que resultou no menor valor de AIC após a análise (Silva et al., 2015a).

Todos os dados obtidos foram avaliados quanto a normalidade e homocedasticidade das variâncias dos tratamentos. As variáveis foram submetidas a análise de regressão, tendo como fator regressor o percentual do Beef Angus ${ }^{\circledR}$. Nesta análise, foi observado a significância dos efeitos linear, quadrático e não significância da falta de ajuste do modelo, sendo a probabilidade de erro tipo I utilizada para tomada de decisão $5 \%$. A variável escore fecal, por tratar-se de uma variável não paramétrica, foi analisada por meio do teste de Kruskal e Wallis (1952) seguido pelo procedimento de Conover (1980) com nível de significância de $5 \%$ de probabilidade de erro tipo I. 
Uso de níveis crescentes de concentrado extrusado e silagem de milho na dieta de ovinos

\section{Resultados e discussão}

A medida com que aumentou a proporção de silagem de milho na dieta houve redução linear no consumo de matéria seca (CMS), CMS em relação ao peso corporal (CMS/PC) e CMS pelo peso metabólico (CMS/PC ${ }^{0,75}$ ), ou seja, as dietas contendo maiores níveis de concentrado extrusado obtiveram maior consumo $(\mathrm{P}<0,05)$ (Tabela 3$)$.
A redução no CMS ocorreu pelo aumento na concentração de fibra nas dietas. De acordo com Macedo Júnior et al. (2012), caso os níveis de fibra da dieta cheguem a comprometer a oferta de carboidratos fermentáveis de origem não fibrosa há redução no CMS. Sendo assim, a maior oferta de silagem de milho que contém maior proporção de fibra levou a redução no consumo das ovelhas.

Tabela 3 - Consumo e digestibilidade da matéria seca em ovelhas consumindo diferentes proporções de concentrado extrusado (Beef Agnus ${ }^{\circledR}$ ) e Silagem de milho

\begin{tabular}{ccccccccc}
\hline & \multicolumn{9}{c}{ Tratamentos } & & & \\
\cline { 2 - 4 } & 70B:30S & 60B:40S & 50B:50S & 40B:60S & P-Valor & MG & CV \\
\hline${ }^{1}$ CMS (kg/dia) & 1,77 & 1,59 & 1,17 & 1,04 & 0,0257 & 1,40 & 20,09 \\
${ }^{2} \mathrm{CMS} /$ PC (\%) & 3,74 & 3,49 & 2,59 & 2,28 & 0,0198 & 3,03 & 14,92 \\
${ }^{3} \mathrm{CMS} /{ }^{\mathrm{PC} 0,75}$ & 98,27 & 90,71 & 67,2 & 59,32 & 0,0074 & 78,89 & 15,43 \\
DMS (\%) & 62,43 & 65,44 & 63,17 & 54,73 & 0,3675 & 61,44 & 16,50 \\
\hline
\end{tabular}

B: Beef Agnus ${ }^{\circledR}$ (concentrado extrusado); S: silagem de milho; CMS: consumo de matéria seca; PC: peso corporal; DMS: digestibilidade da matéria seca; MG: média geral; CV: coeficiente de variação; ${ }^{1} Y=2,570760-0,026008 x, R^{2}=95,91 \% ;{ }^{2} Y=5,413860-0,052958 x, R^{2}=95,00 \% ;{ }^{3} Y=142,037740$ $-1,403202 x, R^{2}=95,23 \%$

Outra explicação para a redução no consumo de matéria é sobre o processamento do concentrado. A extrusão promove sincronismo entre as fontes alimentares e altera a estrutura da matéria prima durante o processo de extrusão, o que facilita a digestão e consequente aumento do consumo (REDDY; REDDY, 2015). Além disso, as partículas de produtos extrusados como demonstrado por Oliveira et al. (2019) têm tamanho próximo a $2 \mathrm{~mm}$, ou seja, são menores e mais homogêneas que as da silagem de milho utilizadas. Partículas menores tem uma maior taxa de passagem sendo necessário um maior consumo com maior inclusão de Beef Agnus ${ }^{\circledR}$.

A recomendação de CMS diário para a categoria animal avaliada é de $1,05 \mathrm{~kg} /$ dia (NRC, 2007), indicando que todos os grupos atingiram a normalidade. $\mathrm{O}$ tratamento 70B:30S está 68,57\% acima da referência, já os animais que consumiram 40B:60S ficou 0,01\% abaixo da mesma. Esses valores reforçam que a maior inclusão de concentrado extrusado promoveu o aumento no CMS. Silva et al. (2020a) e Ruela et al. (2020) também demonstraram aumento no CMS elevados com a utilização de alimentos extrusados na dieta para ovelhas adultas.

Para a digestibilidade da matéria seca (DMS) não houve efeito do aumento na proporção de silagem de milho $(\mathrm{P}>0,05)$ (Tabela 3). Alimentos concentrados possuem maior presença de carboidratos não fibrosos em suas composições, fator que proporciona altas taxas de digestibilidade devido à elevada capacidade de fermentação no rúmen desses componentes (Vallejo-Hernández et al., 2018). Com aumento da proporção de concentrado extrusado embora maior digestibilidade que carboidratos fibrosos tem maior taxa de passagem pelo trato gastrointestinal reduzindo o aproveitamento. Logo, em dietas que aumenta a proporção de silagem de milho, pela maior concentração de fibras e tamanho da partícula permanecem mais tempo no ambiente ruminal expostos para degradação. Essa compensação levou a não significância para digestibilidade entre os tratamentos (Tabela 3).

Não houve efeito das proporções de volumoso e concentrado extrusado sobre o consumo de água $\left(\mathrm{CH}_{2} \mathrm{O}\right)$ e consumo de água em relação ao consumo de matéria seca $\left(\mathrm{CH}_{2} \mathrm{O} / \mathrm{CMS}\right)(\mathrm{P}>0,05)$ (Tabela 4). De acordo com o NRC (2007) o $\mathrm{CH}_{2} \mathrm{O} / \mathrm{CMS}$ diário de ovinos adultos precisa estar entre $2 \mathrm{~L} / \mathrm{kg}$ e $3 \mathrm{~L} / \mathrm{kg}$, portanto, como a média de 2,67 (L/kg), as diferentes relações entre Beef Agnus ${ }^{\circledR}$ e silagem de milho a ingestão hídrica esteve dentro do recomendado para a categoria (Tabela 4).

Da mesma forma que para o consumo de água os parâmetros urinários não apresentaram diferenças estatísticas entre os tratamentos (Tabela 4) $(\mathrm{P}>0,05)$. De acordo com Hendrix (2005) a recomendação de volume de urina (VU) excretado para ovinos, é de 0,1 a $0,4 \mathrm{~L}$ à cada $10 \mathrm{~kg}$ de peso vivo. Como os animais dessa situação experimental tinham peso médio de $50,0 \mathrm{~kg}$, o VU indicado seria entre 0,5 e 2,0, dentro do esperado. A Densidade da Urina (DU) específica de ovinos varia entre 1,020-1,040 g. $\mathrm{mL}^{-1}$ (Hendrix, 2005), portanto, todos os grupos estão dentro da faixa de recomendação. A estabilização nos valores de $\mathrm{VU}$ e DU podem ser relacionados com o $\mathrm{CH}_{2} \mathrm{O}$ adequado, pois segundo Reece (2006) a restrição hídrica pode acarretar em problemas renais e consequentemente, redução no VU e alterações na DU. 
Rodrigues, G. R. D. et al.

Tabela 4 - Ingestão de água, parâmetros fecais e urinários de ovelhas consumindo diferentes níveis de concentrado extrusado (Beef Agnus ${ }^{\circledR}$ ) e silagem de milho

\begin{tabular}{|c|c|c|c|c|c|c|c|}
\hline & \multicolumn{4}{|c|}{ Tratamentos } & \multirow[b]{2}{*}{ P-Valor } & \multirow[b]{2}{*}{ MG } & \multirow[b]{2}{*}{$\mathrm{CV}$} \\
\hline & 70B:30S & 60B:40S & 50B:50S & 40B:60S & & & \\
\hline $\mathrm{CH}_{2} \mathrm{O}(\mathrm{L} / \mathrm{dia})$ & 4,44 & 3,00 & 3,97 & 2,99 & 0,3789 & 3,60 & 31,87 \\
\hline $\mathrm{CH}_{2} \mathrm{O} / \mathrm{CMS}(\mathrm{L} / \mathrm{kg})$ & 2,54 & 1,89 & 3,34 & 2,90 & 0,4725 & 2,67 & 32,51 \\
\hline VU (L/dia) & 1,76 & 0,93 & 1,58 & 1,25 & 0,6987 & 1,38 & 42,8 \\
\hline DU $\left(g \cdot \mathrm{mL}^{-1}\right)$ & 1,0214 & 1,0336 & 1,023 & 1,0216 & 0,7412 & 1,0249 & 0,90 \\
\hline PFMN (kg) & 1,82 & 1,48 & 1,40 & 1,38 & 0,2147 & 1,52 & 22,73 \\
\hline PFMS (g) & 0,624 & 0,533 & 0,461 & 0,562 & 0,2954 & 0,545 & 21,58 \\
\hline MSF (\%) & 36,45 & 37,87 & 34,50 & 40,67 & 0,4125 & 37,35 & 32,90 \\
\hline $\mathrm{EF}^{*}$ & 2,68 & 2,68 & 2,56 & 2,40 & 0,8897 & 2,58 & $\mathrm{XXX}$ \\
\hline
\end{tabular}

B: Beef Agnus ${ }^{\circledR}$ (concentrado extrusado); S: silagem de milho; CMS: consumo de matéria seca; VU: volume urinário; DU: densidade da urina; PFMN: peso das fezes na matéria natural; PFMS: peso das fezes na matéria seca; MSF: matéria seca fecal; EF: escore fecal; MG: média geral; CV: coeficiente de variação; *Estatística não paramética.

Os parâmetros fecais também não diferenciaram com as diferentes proporções de silagem de milho e o volumoso extrusado na dieta das ovelhas $(\mathrm{P}>0,05)$ (Tabela 4). As fezes de ruminantes podem ser influenciadas quantitativamente e qualitativamente pela temperatura ambiental, qualidade alimentar consumida, manejos adotados e características individuais dos animais. $O$ peso do volume fecal excretado pode ser afetado pela taxa de passagem dos alimentos no rúmen e suas digestibilidades (Santos e Nogueira, 2012). De acordo com Vieira (2008), uma ovelha adulta produz entre 0,8 e 1,5 kg de fezes na matéria natural (PFMN), portanto, a média verificada de $1,52 \mathrm{~kg}$ indica que as dietas ofertadas não influenciaram negativamente no PFMF e digestibilidade dos alimentos. A matéria seca fecal (MSF) está dentro da faixa de recomendação de Van Cleef et al. (2010) para ovinos, onde considera-se normal de 37 a $44 \%$ de MSF e a média obtida foi $37,35 \%$. Similar aos encontrado nos estudos com produtos extrusados, Silva et al. (2020b) obtiveram PFMN e MSF de 2,57 kg/dia e 34,60\%, respectivamente. Já Oliveira et al. (2019) constataram 1,798 kg/dia e $31,74 \%$ para as mesmas variáveis. Araújo et al. (2020) verificaram PFMN de 2,47 kg/dia e MSF de 38,89\%.

A avaliação do escore fecal (EF) é fundamental para avaliação de possíveis distúrbios no trato gastrointestinal e seus impactos na saúde e desempenho dos animais. O EF descrito por Gomes et al. (2012) como ideal é 2 , estando a média próxima a este valor com 2,58 . Silva et al. (2020b), Oliveira et al. (2018) e Araujo et al. (2020) avaliando a inclusão de alimentos extrusados para ovelhas, obtiveram EF de 2,47, 3,2 e 2,65, respectivamente, indicando que o uso desses produtos, em diferentes relações concentrado:volumoso, não alteram a digestibilidade dos alimentos e o escore das fezes de ovinos.
Não houve diferença estatística para o tempo gasto em ingestão, ruminação, ócio e mastigação de ovelhas consumindo diferentes proporções de silagem de milho e concentrado extrusado na dieta $(P>0,05)$ (Tabela 5). No entanto, houve redução linear na eficiência ruminação mastigação à medida que aumento a proporção de silagem de milho na dieta $(\mathrm{P}<0,05)$ (Tabela 5).

O aumento na eficiência de ruminação e mastigação pode ter sido proveniente do tamanho médio de $2 \mathrm{~mm}$ das partículas do concentrado extrusado, uma vez são digeridas com maior facilidade pelos microrganismos ruminais, e consequentemente, levam um tempo menor para atingir a dimensão necessária para seguirem ao retículo.

O tempo de ruminação médio diário para ovinos adultos varia de 240 a 540 minutos, podendo ser diretamente influenciado pela natureza das dietas ofertadas e o teor da parede celular contida nos alimentos volumosos (Van Soest, 1994). O único grupo fora dessa referência é o $70 \mathrm{~B}: 30$ S, estando $18,75 \%$ abaixo do valor mínimo. O emprego de forragens na nutrição de ruminantes é de suma importância para a ruminação dos animais, já que a fibra estimula esse processo. Alimentações que reduzem a ruminação animal podem diminuir a produção de saliva, $\mathrm{pH}$ ruminal e digestibilidade da fibra (Geron et al., 2013). Dessa forma, o tratamento 70B:30S pode ter tido menor tempo de ruminação devido à menor presença de fibra na dieta e o tamanho das partículas do concentrado extrusado utilizado (Tabela 5).

Em relação ao metabolismo das ovelhas, a glicemia não alterou significativamente com aumento da silagem de milho na dieta $(P>0,05)$, entretanto, suas concentrações por horários sofreram resposta quadrática $(\mathrm{P}<0,05)$ (Tabela 6). 
Uso de níveis crescentes de concentrado extrusado e silagem de milho na dieta de ovinos

Tabela 5 - Comportamento ingestivo de ovelhas recebendo diferentes relações de concentrado extrusado (Beef Agnus ${ }^{\circledR}$ ) e silagem de milho

\begin{tabular}{|c|c|c|c|c|c|c|c|}
\hline & \multicolumn{4}{|c|}{ Tratamentos } & \multirow[b]{2}{*}{ P-Valor } & \multirow[b]{2}{*}{ MG } & \multirow[b]{2}{*}{ CV } \\
\hline & $70 \mathrm{~B}: 30 \mathrm{~S}$ & 60B:40S & 50B:50S & 40B:60S & & & \\
\hline Ingerindo (min/dia) & 289 & 286 & 201 & 195 & 0,2541 & 242,75 & 30,04 \\
\hline Ruminando (min/dia) & 195 & 255 & 332 & 313 & 0,2478 & 273,75 & 31,70 \\
\hline Ócio (min/dia) & 956 & 899 & 907 & 932 & 0,2698 & 923,50 & 11,91 \\
\hline Mastigação (min/dia) & 484 & 541 & 533 & 508 & 0,3210 & 516,50 & 21,29 \\
\hline Eficiência (Ing) (g/min) & 6,74 & 5,67 & 5,95 & 6,12 & 0,1254 & 6,12 & 37,97 \\
\hline Eficiência $(\text { Rum })^{1}(\mathrm{~g} / \mathrm{min})$ & 9,75 & 8,46 & 3,87 & 3,51 & 0,0025 & 6,40 & 34,11 \\
\hline Eficiência (Mast) ${ }^{2}(\mathrm{~g} / \mathrm{min})$ & 3,79 & 3,00 & 2,31 & 2,19 & 0,0003 & 2,82 & 28,15 \\
\hline
\end{tabular}

B: Beef Agnus ${ }^{\circledR}$ (concentrado extrusado); S: silagem de milho; Ing: ingestão; Rum: ruminação; Mast: Mastigação; MG: média geral; CV: coeficiente de variação; ${ }^{1} \mathrm{Y}=16,895620-0,233146 \mathrm{x}, \mathrm{R}^{2}=89,95 \%$; ${ }^{2} \mathrm{Y}=5,299320-0,054926 \mathrm{x}, \mathrm{R}^{2}=92,60 \%$

Tabela 6 - Concentração glicêmica por tratamento e horário de coleta de ovinos alimentados com diferentes relações de Beef Agnus ${ }^{\circledR}$ e Silagem de milho

\begin{tabular}{|c|c|c|c|c|c|c|c|}
\hline & \multicolumn{5}{|c|}{ Tratamentos } & \multirow[b]{2}{*}{ MG } & \multirow[b]{2}{*}{ CV } \\
\hline & \multicolumn{2}{|c|}{ 70B:30S } & 60B:40S & 50B:50S & 40B:60S & & \\
\hline Glicemia (mg/dL)* & \multicolumn{2}{|c|}{50,48} & 62,56 & 52,60 & 58,40 & 56,07 & 12,05 \\
\hline \multicolumn{8}{|c|}{ Horários de coleta } \\
\hline & 08h & $11 \mathrm{~h}$ & $14 \mathrm{~h}$ & $17 \mathrm{~h}$ & $20 \mathrm{~h}$ & P-valor & P-valor* \\
\hline Glicemia $^{1}(\mathrm{mg} / \mathrm{dL})$ & 61,50 & 55,60 & 49,00 & 54,40 & 59,85 & 0,0458 & 0,1258 \\
\hline
\end{tabular}

MG: média geral; CV: coeficiente de variação; ${ }^{1} \mathrm{Y}=107,190635-7,861111 \mathrm{x}+0,275397 \mathrm{x}^{2}, \mathrm{R}^{2}=90,97 \%$; * corresponde ao P-valor da glicemia entre os tratamentos.

O mesmo perfil glicêmico entre os tratamentos (Tabela 6) foi provocada pela pouca variação que os teores desse açúcar sofrem no plasma sanguíneo, uma vez que há intenso controle hormonal desse metabólito pelas ações da insulina e glucagon sobre o glicogênio e glicorticoides durante a gliconeogênese (Silva et al., 2020a). Ainda de acordo com Silva et al. (2020a) o valor de referência de glicose para ovinos é de 30 - $94 \mathrm{mg}$ $\mathrm{dL}^{-1}$, portanto, todos os tratamentos apresentaram-se com números adequados, indicando que as diferentes porcentagens ofertadas do concentrado extrusado não prejudicaram as concentrações de glicose dos animais, durante o período experimental.

Com relação à glicemia por horário, a diferença ocorreu devido ao perfil de degradação dos alimentos e aos horários de alimentação (Tabela 6) $(\mathrm{P}<0,05)$. A menor concentração de glicose verificada foi no horário das 14:00 horas. Os carboidratos solúveis são fermentados no rúmen, entretanto, dependendo do processamento em que os grãos dos cereais que compõem a dieta são submetidos, parte desses compostos escapam da fermentação ruminal e são digeridos no intestino delgado pelas enzimas de origem pancreáticas ( $\alpha$-amilase) e mucosa intestinal (maltase e isomaltase) (Cañizares et al., 2009). Sendo assim, a parede intestinal pode ter roubado parte da glicose ingerida e ocasionado a resposta quadrática verificada para essa variável.

Já o aumento às 17:00 e 20:00 horas pode ter sido ocasionado pela lenta ação da gliconeogênese, que utiliza uma via indireta e ocorre de forma lenta. O propionato é transformado em propionil CoA, metimalonil CoA, succinil CoA, passa pelo ciclo de Krebs como succionato e em seguida chega à veia porta hepática como glicose (Kozloski, 2017). Portanto, os carboidratos solúveis na dieta podem ter levado a um incremento na produção de ácido propiônico, e consequentemente, aumentado a concentração de glicose plasmática dos animais durante às 17:00 e 20:00 horas.

Para os metabolitos proteicos e energéticos não houve efeito das diferentes relações concentrado: volumoso utilizadas $(\mathrm{P}>0,05)$ (Tabela 7). Os triglicerídeos são sintetizados no fígado e atuam como a principal forma de armazenamento de ácidos graxos no tecido adiposo, constituindo fontes de reserva de energia. Seus níveis encontrados no plasma refletem o fornecimento de energia proveniente da dieta consumida, assim como sua qualidade. Já o colesterol apresenta origem exógena e endógena, sendo a primeira pela alimentação e a segunda pela síntese realizada no intestino delgado, células 
Rodrigues, G. R. D. et al.

adiposas e fígado. Esse metabólito é um indicador dos níveis lipídeos presentes na corrente sanguínea (Kozloski, 2017). Uma vez que os valores obtidos estão dentro da referência de Silva et al. (2020a), as dietas ofertadas não comprometeram o metabolismo energético dos animais e consequentemente, proporcionaram armazenamento de energia adequado aos animais.

Tabela 7 - Concentração dos metabólitos energéticos e proteicos em função das diferentes relações concentrado extrusado (Beef Agnus ${ }^{\circledR}$ ) e Silagem de milho

\begin{tabular}{|c|c|c|c|c|c|c|c|c|}
\hline \multicolumn{9}{|c|}{ Tratamentos } \\
\hline & $70 \mathrm{~B}: 30 \mathrm{~S}$ & 60B:40S & 50B:50S & 40B:60S & \multirow[t]{2}{*}{ MG } & \multirow[t]{2}{*}{ P-valor } & \multirow[t]{2}{*}{ CV } & \multirow[t]{2}{*}{$\mathrm{VR}^{* *}$} \\
\hline \multicolumn{5}{|c|}{ Metabólitos Energéticos } & & & & \\
\hline Triglicerídeos (mg/dL) & 60,50 & 62,66 & 70,12 & 69,10 & 65,35 & 0,8708 & 32,36 & $5-71$ \\
\hline Colesterol (mg/dL) & 14,20 & 16,13 & 15,41 & 15,13 & 15,21 & 0,8936 & 26,13 & $14-126$ \\
\hline \multicolumn{9}{|c|}{ Metabólitos Proteicos } \\
\hline Ureia (mg/dL) & 43,46 & 34,26 & 34,58 & 31,46 & 36,01 & 0,4154 & 32,07 & $10-92$ \\
\hline $\mathrm{PT}^{* 1}(\mathrm{mg} / \mathrm{dL})$ & 4,32 & 4,01 & 4,87 & 4,76 & 4,47 & 0,0823 & 11,66 & $3,1-10,7$ \\
\hline Ácido úrico (mg/dL) & 0,05 & 0,04 & 0,07 & 0,17 & 0,08 & 0,1870 & 4,37 & $0-1,7$ \\
\hline Albumina (mg/dL) & 3,41 & 2,97 & 3,64 & 3,36 & 3,33 & 0,6287 & 23,00 & $1,1-5,2$ \\
\hline Creatinina (mg/dL) & 0,60 & 0,61 & 0,60 & 0,63 & 0,61 & 0,9700 & 17,95 & $0,4-1,7$ \\
\hline
\end{tabular}

PT*: Proteínas Totais; MG: média geral; CV: coeficiente de variação; P: probabilidade de significância (5\%); VR**: Valor de referência de Silva et al. (2020a); ${ }^{1} \mathrm{X}=7,182812-0,078172 \mathrm{x}+0,000512 \mathrm{x}^{2}, \mathrm{R}^{2}=52,90 \%$

Com relação ao metabolismo proteico, a variável proteína total (PT) apresentou resposta quadrática em função da inclusão de silagem de milho. As PT participam do transporte de nutrientes, metabólitos e hormônios, regulam o pH sanguíneo e participam da coagulação sanguínea. Sua síntese está diretamente relacionada com o status nutricional do animal e é realizada principalmente no fígado (Kozloski, 2017).

Já a albumina (Tabela 7) é um constituinte de grande parte da proteína sérica total, sendo responsável por compor reservas energéticas e transportar ácidos graxos livres no organismo. Como esse metabólito também está na referência de Silva et al. (2020a), seus índices reforçam que os resultados da variável PT não promoveram distúrbios metabólicos.

Não foram encontradas diferenças estatísticas $(\mathrm{P}>0,05)$ para a ureia e ácido úrico. A ureia é o principal indicador do nível proteico em ruminantes, já que tem relação estreita com a digestão proteica e metabolismo da microbiota ruminal. A amônia absorvida pela parede do rúmen é transportada pelo fígado e metabolizada em ureia. O ácido úrico apresenta ação direta no metabolismo ruminal, sendo seus níveis influenciados pela qualidade dos alimentos ingeridos e produção de proteína ruminal (Kozloski, 2017). Como essas duas variáveis se apresentaram no intervalo de referência de Silva et al. (2020a), os alimentos ofertados nesse estudo possuíam ampla qualidade nutricional e não inferiram de modo negativo na formação de proteína microbiana no rúmen.

Com relação a creatinina, não foram verificadas diferenças estatísticas $(\mathrm{P}>0,05)$ e seus índices encontram-se no valor de referência de Silva et al. (2020a). Esse metabólito proteico é importante indicativo da função renal, uma vez que reflete a taxa de filtração glomerular e sofre pouca influência de fatores intrínsecos e extrínsecos aos animais, como dieta, idade ou sexo (Reece, 2006).

\section{Conclusão}

O aumento em até $60 \%$ de silagem na dieta e 40\% de concentrado extrusado (dieta 40B:60S) leva a redução do consumo de matéria seca, da eficiência de mastigação e de ruminação. No entanto, não altera a digestibilidade da matéria seca, o consumo de água, os parâmetros urinários, fecais e metabolitos. Ou seja, o uso de dietas entre $40 \%$ a $70 \%$ de volumoso extrusado pode ser utilizado na dieta de ovinos sem levar a prejuízos no aproveitamento das dietas e no metabolismo.

\section{Aprovação do Comitê de Ética}

O ensaio contou com a aprovação da Comissão de Ética e Utilização dos Animais (CEUA) da Universidade Federal de Uberlândia sob o número de protocolo 092/16. 
Uso de níveis crescentes de concentrado extrusado e silagem de milho na dieta de ovinos

\section{Referências}

Araujo, C. M., Oliveira, K. A., Macedo Junior, G. L., Silva, S. P., Silva, D. A. P. 2020. Parâmetros nutricionais e bioquímicos de ovinos consumindo volumoso extrusado com diferentes teores de Uruchloa brizantha em comparação a silagem de milho tradicional. Caderno De Ciências Agrárias. 12: 1-11. Doi: https://doi.org/10.35699/24476218.2020.25810.

Cañizares, G. I. L., Rodrigues, L., Cañizares, M. C. 2009. Metabolismo de carboidratos não-estruturais em ruminantes. Archives of Veterinary Science. 14 (1): 63-73. Doi: https://doi.org/10.5380/avs.v14i1.13615.

Conover, W. J. 1980. Practical Nonparametric Statistics. 2nd Edition, John Wiley \& Sons, New York.

Fischer, V.; Deswysen, A. G.; Dèspres, L.; Dutilleul, P.; Lobato, J. F. P. 1998. Padrões nectemerais do comportamento ingestivo de ovinos. Revista Brasileira de Zootecnia. 27: 362-369.

Geron, L. J. V., Mexia, A. A., Cristo, R. L., Garcia, J., Cabral, L. S., Trautmann, R. J., Martins, O. S., Zeoula, L. M. 2013. Consumo, digestibilidade dos nutrientes e características ruminais de cordeiros alimentados com níveis crescentes de concentrado em ambiente tropical no Vale do Alto Guaporé - MT. Semina: Ciências Agrárias, 34 (5): 2497-2510. doi: https://doi.org/10.5433/1679-0359.2013v34n5p2497.

Gomes, S. P.; Borges, I.; Borges, A. L. C. C.; Macedo Junior, G. L.; Campos, W. E.; Brito, T. S. 2012. Tamanho de partícula do volumoso e frequência de alimentação sobre o metabolismo energético e proteico em ovinos, considerando dietas com elevada participação de concentrado. Revista Brasileira de Saúde e Produção Animal. 13: 732-744. Doi: https://doi. org/10.1590/S1519-99402012000300013.

Hendrix, C. M. 2005. Procedimentos laboratoriais para técnicos veterinários (4th ed.). São Paulo: Rocca, 556p.

Kozloski, G. V. 2017. Bioquímica dos ruminantes (3rd ed.). Rio Grande do Sul: editora UFSM, 203p.

Kruskal, W. H.; Wallis, W. A. 1952. Use of ranks in one-criterion variance analysis. Journal American Statistical Association. 47:583-621. Doi: https://doi.org/10.1080/01621459.1952.10483441.

Macedo Junior, G. D. L., Ruela, P. A. C., Oliveira, K. A., Araújo, C. M., Varanis, L. F. M., \& Assis, T. S. D. 2019. Avaliação do desempenho e comportamento ingestivo de ovinos recebendo ração extrusada em diferentes relações volumoso e concentrado. Vet. Not., 144-160. doi: http://dx.doi.org/10.14393/VTN-v25n2-2019-38920.

Macedo Júnior, G. L, Sousa, L. F., Godoi, F. N., Perez, J. R. O., França, P. M., Almeida, T. R. V., Paula. O. J., Assis, R. M. 2012. Consumo, digestibilidade aparente e balanço de nitrogênio em ovelhas alimentadas com diferentes níveis de fibra em detergente neutro. Ciência Animal Brasileira, 13(1): 33-40. Doi: https://doi.org/10.5216/cab.v13i1.9481.

Maynard, D. G.; Stewart, J. W. B.; Bettany, J. R. 1984. Sulfur cycling in grassland and parkland soils. Biogeochemistry. 1: 97-111. Doi: https:// doi.org/10.1007/BF02181123.

Mourão, R. C., Pancoti, C. G., Moura, A. M., Ferreira A. L., Borges, A. L. C. C., Silva, R. R. 2012. Processamento do milho na alimentação de ruminantes. PUBVET, 6 (5): 1-27. Doi: https://doi.org/10.22256/ pubvet.v16n5.1292.

National Research Council - NRC. 2007. Nutrient Requeriments of Small Ruminants. 1. ed. Washington, DC, USA.

Oetzel, G. R. 2017. Diagnosis and management of subacute ruminal acidosis in dairy herds. Vet Clin Food Animal, 33: 463-480. Doi: https:// doi.org/10.1016/j.cvfa.2017.06.004.
Oliveira, K. A; Macedo Junior, G. L; Silva, S. P; Araújo, C. M; Varanis, L. F. M; Sousa, L. F. 2019. Nutritional and metabolic parameters of sheep fed with extrused roughage in comparison with corn silage. Semina: Ciências Agrárias. 39: 1795-1804. Doi: https://doi.org/10.5433/16790359.2018v39n4p1795.

Reddy, G.V.N. \& Reddy, Y. R. 2015. Extrusion Technology. In: Kundu, S.S., S.K. Mahanta, S. Singh, and P.S. Pathak (eds). Animal Feed Technology. Satish Serial Publishing House, New Delhi. Pp. 311-326.

Reece, W. O. 2006. Função Renal nos Mamíferos. In: REECE, W. O. DUKES - Fisiologia dos animais domésticos. 12. ed. Rio de Janeiro, Guanabara Koogan. p. 68-96.

Ruela, P. A. C., Oliveira, K. A., Sousa, L. M., Silva, S. P., Sousa, L. F, Macedo Júnior, G. L. 2020. Consumo, comportamento ingestivo e perfil metabólico de ovinos alimentados com ração extrusada com diferentes relações volumoso: concentrado. Caderno de ciências agrárias, 12: 01-08. Doi: https://doi.org/10.35699/2447-6218.2020.20412.

Santos, F. M., Araújo, G. G. L., Souza, L. L., Yamamoto, S. M., Queiroz, M. M. A., Lanna, D. P. D., Moraes, S. A. 2019. Impact of water restriction periods on carcass traits and meat quality of feedlot lambs in the Brazilian semi-arid region. Meat Science, 156: 196-204. Doi: https:// doi.org/10.1016/j.meatsci.2019.05.033.

Santos I. A., Nogueira L. A. H. 2012. Estudo energético do esterco bovino: seu valor de substituição e impacto da biodigestão anaeróbia. Revista Agrogeoambiental, 4 (1): 41-49. Doi: http://dx.doi.org/10.18406/2316$1817 v 4 n 12012373$.

SAS Institute Inc, JMP ${ }^{\circledR} 10$ Modeling and Multivariate Methods. 2012. Cary, NCSAS Institute Inc.

Silva, D. A. P., Varanis, L. F. M., Oliveira, K. A., Sousa, L. M., Siqueira, M. T. S., Macedo Júnior, G. L. 2020a. Parâmetros de metabólitos bioquímicos em ovinos criados no Brasil. Caderno de ciências agrárias, 12: 01-08. Doi: https://doi.org/10.35699/2447-6218.2020.20404.

Silva, D. A. P., Santana, A. G., Araújo, C. M., Oliveira, K. A., Siqueira, M. T. S., Macedo Júnior, G. L. 2020b. Avaliação dos efeitos nutricionais e metabólicos da substituição de silagem de milho por ração extrusada de Capim-marandu (Urochloa brizantha) em ovinos. Caderno de ciências agrárias, 12: 01-09. Doi: https://doi.org/10.35699/24476218.2020.19833.

Silva, E. N.; Duarte, J. B.; Reis, A. J. 2015a. Seleção da matriz de variânciacovariância residual na análise de ensaios varietais com medidas repetidas em cana-de-açúcar. Ciência Rural 45: 993-999. Doi: http:// dx.doi.org/10.1590/0103-8478cr20141531.

Silva, M. F. L., Santos, L., Choupina, A. 2015b. A extrusão em tecnologia alimentar: tipos, vantagens e equipamentos. Revista de Ciências Agrárias, 38 (1): 3-10.

Singh, J.; Dartois, A. e Kaur, L. 2010. Starch digestibility in food matrix: a review. Trends in Food Science \& Technology, 21 (4): 168-180. Doi: https://doi.org/10.1016/j.tifs.2009.12.001.

Valadares Filho, S. C.; Lopes, S.A.; Machado, P.A.S.; Chizzotti, M.L; Amaral, H. F.; Magalhães, K.A.; Rocha Junior, V. R.; Capelle, E.R. 2018. CQBAL 4.0. Tabelas Brasileiras de Composição de Alimentos para Bovinos. Disponível em: www.ufv.br/cqbal.

Vallejo-Hernández, L. H.; Elghandour, M. M. Y.; Greiner, R.; Anele, U. Y.; Rivas-Cáceres, R. R.; Barros-Rodríguez, M.; Salem, A. Z. M. 2018. Environmental impact of yeast and exogenous xylanase on mitigating carbon dioxide and enteric methane production in ruminants. Journal of Cleaner Production, 189: 40-46. Doi: https://doi.org/10.1016/j. jclepro.2018.03.310. 
Rodrigues, G. R. D. et al.

Van Cleef, E. H. C. B., Ezequiel J. M. B., D’Aurea A. P., Fávaro, V. R., Sancanari, J. B. D. 2010. Crude glycerin in diets for feedlot Nellore cattle. Revista Brasileira de Zootecnia. 43 (2): 86-91. Doi: https://doi. org/10.1590/S1516-35982014000200006.

Van Soest, P. J. 1994. Nutritional ecology of the ruminant. 2.ed. Ithaca: Cornell University Press. Doi: https://doi.org/10.7591/9781501732355.
Vieira L. S. 2008. Métodos alternativos de controle de nematóides gastrintestinais em caprinos e ovinos. Embrapa Caprinos e Ovinos-Artigo em periódico indexado. Disponível em: https:// www.alice.cnptia.embrapa.br/bitstream/doc/533824/1/ APIMetodosalternativosdecontroledenematoides.pdf. Acesso em: 09 de nov. 2020. 